\title{
Investigations of Slip Effect on the Performance of Micro Gas Bearings and Stability of Micro Rotor-Bearing Systems
}

\author{
Hai Huang*, Guang Meng, and Jieyu Chen \\ State Key Laboratory of Mechanical System and Vibration, Shanghai Jiao Tong University, 800 \\ DongChuan Road, Shanghai, 200240, People Republic of China); \\ E-mails: huanghai2003@gmail.com; gmeng@sjtu.edu.cn
}

* Author to whom correspondence should be addressed: huanghai2003@ gmail.com

Received: 27 June 2007 / Accepted: 2 August 2007 / Published: 3 August 2007

\begin{abstract}
Incorporating the velocity slip effect of the gas flow at the solid boundary, the performance and dynamic response of a micro gas-bearing-rotor system are investigated in this paper. For the characteristic length scale of the micro gas bearing, the gas flow in the bearing resides in the slip regime rather than in the continuum regime. The modified Reynolds equations of different slip models are presented. Gas pressure distribution and load carrying capacity are obtained by solving the Reynolds equations with finite different method (FDM). Comparing results from different models, it is found that the second order slip model agrees reasonably well with the benchmarked solutions obtained from the linearized Boltzmann equation. Therefore, dynamic coefficients derived from the second order slip model are employed to evaluate the linear dynamic stability and vibration characteristics of the system. Compared with the continuum flow model, the slip effect reduces dynamic coefficients of the micro gas bearing, and the threshold speed for stable operation is consequently raised. Also, dynamic analysis shows that the system responses change with variation of the operating parameters including the eccentricity ratio, the rotational speed, and the unbalance ratio.
\end{abstract}

Keywords: MEMS, Micro rotor, Micro gas bearing, Velocity slip, Mechanical performance, Stability, Dynamical response. 


\section{Introduction}

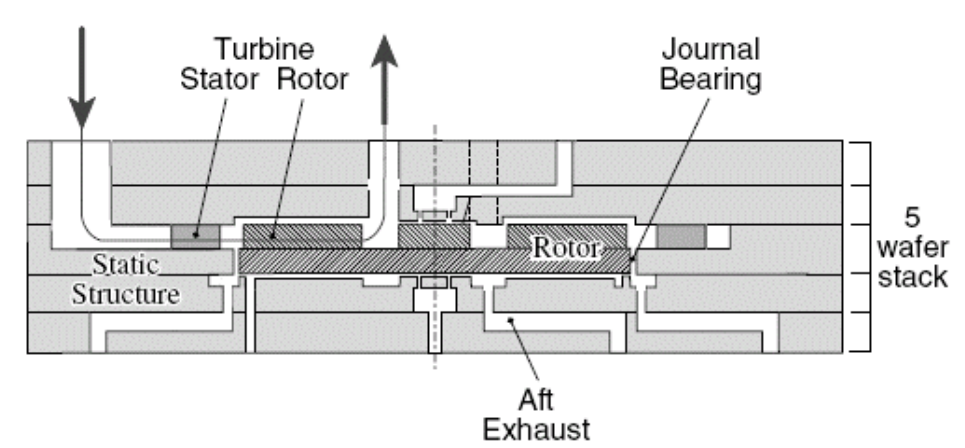

Figure 1. Schematic cross-section of the micro-motor integrated with gas bearing.

In an attempt to address the ever rising demand for high performance compact power source, a new branch of micro-electro-mechanical system called power MEMS has recently been defined ${ }^{[1,2]}$. Among them, the nascent project to develop micro scale gas turbine generators at MIT is specifically targeted for high power density applications. These machines are supported by gas bearings, as shown in Fig.1, and able to achieve rotation rate of more than one million RPM (revolutions per minute) ${ }^{[2]}$. The micro rotor and the micro bearing are fabricated by micro fabrication technology. Different from the traditional rotor system, the micro rotor and the bearing are made of Silicon.

Operating at such high speed, an accurate assessment of the vibration characteristics of the micro rotor system must be made so as to design highly reliable rotary machines and avoid occurrence of dangerous sliding wear and rub impact effect. Thus, the high speed rotor dynamic characteristic of the micro rotor-bearing system is a major concern in the mechanical design of micro motors and such kind of power MEMS. There are many dynamic phenomena and nonlinear problems that can be met for MEMS that may challenge successful operation of these machines. Some researches, which aimed at analyzing and simulating the rotor dynamics in micro rotating machinery ${ }^{[3,4]}$, have been done during the past two decades. Piekos ${ }^{[5]}$ developed a pseudo-spectral method to facilitate orbit simulation of the rotor. Savoulides ${ }^{[6]}$ constructed a small-scale gas bearing model for the rotor. Wang $^{[7]}$ analyzed the bifurcation of a rotor supported by a self-acting gas journal bearing, and the analysis focused on the dynamic behavior of the rotor-bearing system. In these researches, the lubricated gas film was mostly treated as continuum flow, and effects of the micro fluid mechanics have not been considered.

One of the most interesting characteristics in micro rotor-bearing systems is that the nature of the lubricated gas flow departs from the continuum flow regime. For a micro gas bearing, the thickness of the gas film is comparable with the gas molecular mean free path. At this scale, the gas layer adjacent to the surface does not satisfy the no-slip velocity boundary condition. Some researchers have made contributions and improvements to this field. Burgdofer ${ }^{[8]}$ introduced the first-order slip velocity model and derived the modified Reynolds equation. $\mathrm{Hsia}^{[9]}$ and Mitsuya ${ }^{[10]}$ presented the second-order slip model and the 1.5-order modified Reynolds equation. Huang ${ }^{[11]}$ discussed the second-order slip effect on the micro gas bearing steady-state operation performance. Y.H Sun ${ }^{[12]}$ presented analytical investigations of slip flow between the flying head and disk in the hard disk drive. However, these researchers mainly focused on the micro fluid mechanics, and few attentions have been paid to the performance of the micro journal bearing. 
In this study, the performance of the micro bearing is evaluated and the stability of the micro rotor system is discussed. The gas velocity slip effect on the solid boundary is taken into account, and the modified Reynolds equation is solved with the finite difference method (FDM). By comparison of performances of the micro gas bearing such as flow rate, gas pressure distribution, load carrying capacity and attitude angle derived from different models, the slip effect on the performances of the bearing is discussed and the second order slip model is chosen to evaluate dynamic characteristics of the system. The linear dynamic coefficients are employed to acquire the linear threshold speed for stable operation, and the dynamic responses of different speed regimes are compared. It is found that, for a micro gas bearing, the velocity slip effect would result in smaller dynamic coefficients. As a result, the stability of the micro rotor system is compromised and the threshold speed for stable operation is pushed upwards.

\section{Slip model and Reynolds equation}

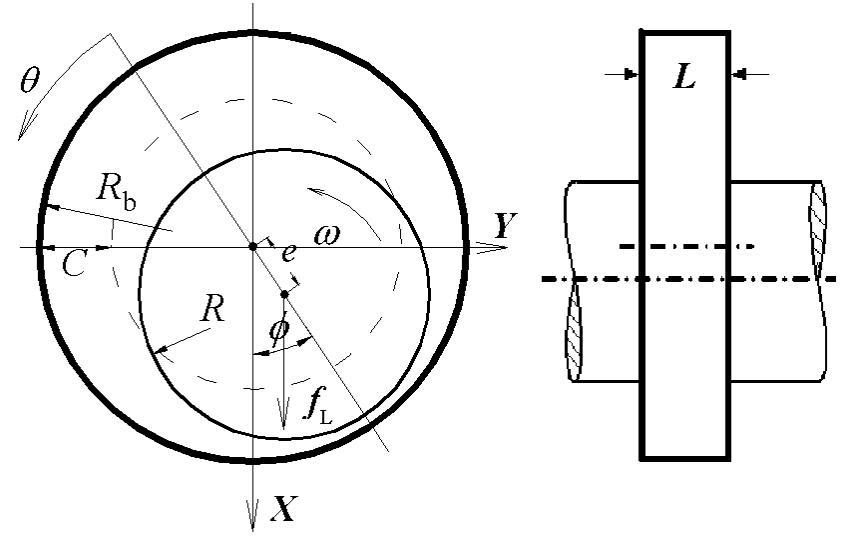

Figure 2. Schematic of the journal bearing in the micro rotor bearing system (not to scale).

Fig. 2 shows the schematic of a micro gas journal bearing to be investigated. The micro rotor spins at an angular velocity of $\omega$ inside a stationary bushing. The bearing's radius is $R_{b}$, and the rotor's radius $R$. When the rotor's center coincides with the bearing's center, the distance between the outer edge of the rotor and the inner edge of the bushing defines the average clearance, $C$. The ratio of the clearance to the radius is denoted by $\psi(\psi=C / R)$. As is shown here in cross sectional view, the bearing is $L$ in length with both ends either exposed to the ambient or treated with a uniform pressure distribution, denoted by $P_{a}$. Due to constraints dictated by MEMS fabrication technologies, the dimension of the gas journal bearing is chosen as $R=2 \mathrm{~mm}, L=300 \mu \mathrm{m}, C=12 \mu \mathrm{m}$, from which we could see that the micro journal bearing is very short compared to their macro-scale counterparts. The bearing's length to diameter ratio is 0.075 , which is at least one order of magnitude smaller than that in conventional gas bearings. This variation inherently alters the driving flow mechanisms in the micro bearing and consequently the performance of the micro bearing and the stability of the micro rotor system.

The mass of the rotor is denoted by $m$, and $e_{m}$, known as unbalance eccentricity, defines the distance between the center of mass and center of geometry of the rotor. The external radial force, denoted by $f_{L}$, exerts on the rotor and pushes the journal center away from the bearing center. The distance between the two centers, known as the eccentricity and denoted by $e$, is usually normalized by the average clearance and expressed as an "eccentricity ratio" denoted by $\varepsilon(\varepsilon=e / C)$. A coordinate 
system is defined such that the $X$-axis is parallel to the direction of the applied force, while the $Y$-axis is perpendicular to the applied force. The angle between the $X$-axis and the line connecting the rotor center and the bearing center defines the attitude angle and is denoted by $\phi$.

In view of the length scale of the micro bearing, the thickness of the film would approach $0.1 \mu \mathrm{m}$ or less. Thus the characteristic length of the gas film is comparable to the mean free path of the gas molecule, and the flow regime could be determined by the Knudsen number, which is defined as $\mathrm{Kn}=\lambda / h$. Here, $\lambda$ is the molecular mean free path, and $h$ the characteristic length of the gas film. According to the category of Bird $^{[13]}$, the gas flow in the bearing resides in the slip regime $\left(10^{-3}<\mathrm{Kn}<0.1\right)$, in which the velocity slip occurs at the solid boundary and the traditional Reynolds equation needs to be modified. If we adopt the first-order slip model, the flow slippage on the surface of the journal and bushing is given as ${ }^{[8]}$

$$
u_{\text {slip }}=-\frac{2-\sigma}{\sigma} \lambda \frac{\partial u}{\partial z}=-a \lambda \frac{\partial u}{\partial z}
$$

where $\sigma$ is the accommodation coefficient relative to momentum, $a=(2-\sigma) / \sigma$ the surface correcting coefficient. When the second-order slip model is taken, the flow slippage becomes ${ }^{[9]}$

$$
u_{\text {slip }}=a \lambda \frac{\partial u}{\partial z}-\frac{\lambda^{2}}{2} \frac{\partial^{2} u}{\partial z^{2}}
$$

And for the 1.5-order slip model, the flow slippage yields ${ }^{[10]}$

$$
u_{\text {slip }}=-a \lambda \frac{\partial u}{\partial z}-\frac{1}{2}\left(\frac{2}{3} \lambda\right)^{2} \frac{\partial^{2} u}{\partial z^{2}}
$$

Using the non-dimensional parameters expressed in Eq.(4), the first order modified Reynolds equation, the second order modified Reynolds equation and the 1.5 order modified Reynolds equation can be written in the non-dimensional form as Eq. (5), Eq. (6) and Eq. (7).

$$
\begin{gathered}
t=\omega \tau, P=p_{a} p, x=R \theta, z=R \xi, H=C h \\
\frac{\partial}{\partial \theta}\left[\left(P H^{3}+6 a \mathrm{~K}_{\mathrm{n}} H^{2}\right) \frac{\partial p}{\partial \theta}\right]+\frac{\partial}{\partial \xi}\left[\left(P H^{3}+6 a \mathrm{~K}_{\mathrm{n}} H^{2}\right) \frac{\partial p}{\partial \xi}\right]=\Lambda \frac{\partial(P H)}{\partial \theta}+2 \Lambda \frac{\partial(P H)}{\partial \tau} \\
\frac{\partial}{\partial \theta}\left[\left(P H^{3}+6 a \mathrm{~K}_{\mathrm{n}} H^{2}+6 \mathrm{~K}_{\mathrm{n}}{ }^{2} \frac{H}{P}\right) \frac{\partial p}{\partial \theta}\right] \\
+\frac{\partial}{\partial \xi}\left[\left(P H^{3}+6 a \mathrm{~K}_{\mathrm{n}} H^{2}+6 \mathrm{~K}_{\mathrm{n}}{ }^{2} \frac{H}{P}\right) \frac{\partial p}{\partial \xi}\right]=\Lambda \frac{\partial(P H)}{\partial \theta}+2 \Lambda \frac{\partial(P H)}{\partial \tau}
\end{gathered}
$$




$$
\begin{aligned}
& \frac{\partial}{\partial \theta}\left[\left(P H^{3}+6 a \mathrm{~K}_{\mathrm{n}} H^{2}+\frac{8}{3} \mathrm{~K}_{\mathrm{n}}{ }^{2} \frac{H}{P}\right) \frac{\partial p}{\partial \theta}\right] \\
& +\frac{\partial}{\partial \xi}\left[\left(P H^{3}+6 a \mathrm{~K}_{\mathrm{n}} H^{2}+\frac{8}{3} \mathrm{~K}_{\mathrm{n}}{ }^{2} \frac{H}{P}\right) \frac{\partial p}{\partial \xi}\right]=\Lambda \frac{\partial(P H)}{\partial \theta}+2 \Lambda \frac{\partial(P H)}{\partial \tau}
\end{aligned}
$$

Here, $\tau, p, \theta, \xi$ and $h$ is the dimensionless time, dimensionless pressure, circumferential coordinate, axial coordinate and film thickness respectively. Where $\Lambda=\frac{6 \mu \omega}{p_{a} \psi^{2}}$ is the bearing number, $\mu$ and $p_{a}$ viscosity and pressure of the ambient gas respectively.

\section{Steady-state performance}

In this section, the steady-state performance of the bearing is studied. Results obtained from the continuum flow, the first-order slip model, the second-order slip model and the 1.5 order slip model are compared with each other and benchmarked against those of the linearized Boltzmann equation[10]. For the micro gas bearing presented, the geometry and environment parameters are list in Tab.1.

Table 1. The geometry and the environment parameters

\begin{tabular}{lcc}
\hline \multicolumn{1}{c}{ Descriptions } & Parameters & Value \\
\hline Radius of the rotor & $R$ & $2 \mathrm{~mm}$ \\
Length of the rotor and the & $L$ & $300 \mu \mathrm{m}$ \\
bearing & $C$ & $12 \mu \mathrm{m}$ \\
Average clearance & $\mathrm{Si}$ & $2.33 \mathrm{~g} / \mathrm{cm}^{3}$ \\
Material of the rotor & $\rho$ & $1.8 \times 10^{-5} \mathrm{~Pa} \cdot \mathrm{s}$ \\
$\quad$ Density & $\mu$ & $1.01325 \mathrm{~Pa}$ \\
Material of the gas & $p_{a}$ & \\
$\quad$ Viscosity & & \\
Environment pressure &
\end{tabular}

The non-dimensionalized flow rates are given by Mitsuya ${ }^{[10]}$ and $\operatorname{Sun}^{[12]}$ as follows:

Continuum flow:

$$
Q_{c}(D)=\frac{D}{6}
$$

First-order slip flow:

$$
Q_{1}(D)=\frac{a \sqrt{\pi}}{2}+\frac{D}{6}
$$

Second-order slip flow:

$$
Q_{2}(D)=\frac{\pi}{4 D}+\frac{a \sqrt{\pi}}{2}+\frac{D}{6}
$$


1.5-order slip flow:

$$
Q_{1.5}(D)=\frac{\pi}{9 D}+\frac{a \sqrt{\pi}}{2}+\frac{D}{6}
$$

where $D=\frac{\sqrt{\pi}}{2 K_{n}}$ is called the inverse Knudsen number. The analytical expressions of the linearized Boltzmann equation is given by Cercignani $^{[14]}$ as follow

$$
Q_{B}(D)=-\frac{1}{D}+\frac{1}{\Delta}\left(C_{11}-\frac{D^{2}}{6} C_{12}+\frac{D^{4}}{144} C_{12}\right)
$$

where the coefficients are ${ }^{[14]}$

$$
\begin{aligned}
C_{11}= & \frac{1}{\sqrt{\pi}}\left\{8-\frac{\sqrt{\pi}}{12} D^{3}+\frac{D^{4}}{16}-2 D\left(4+D^{2}\right) T_{0}(D)\right. \\
& \left.-\left(16+8 D^{2}+\frac{D^{4}}{8}\right) T_{1}(D)-D\left(16+D^{2}\right) T_{2}(D)\right\} \\
C_{22}= & \frac{1}{\sqrt{\pi}}\left\{1-2 T_{1}(D)\right\} \\
C_{12}= & \frac{1}{\sqrt{\pi}}\left\{2-\frac{\sqrt{\pi}}{2} D+\frac{D^{2}}{4}-2 D T_{0}(D)-\left(4+\frac{D^{2}}{2}\right) T_{1}(D)-2 D T_{2}(D)\right\} \\
\Delta= & C_{11} C_{22}-C_{12}^{2}
\end{aligned}
$$

$T_{n}(x)$ is the Abramowitz function and defined as

$$
T_{n}(x)=\int_{0}^{\infty} t^{n} \cdot \exp \left(-t^{2}-\frac{x}{t}\right) d t
$$


Solving the Reynolds equations with finite difference method, the gas pressure of the gas film is obtained. Integrating the dimensionless pressure on the surface of the journal, the dimensionless bearing force components along the radial direction $F_{\mathrm{r}}$ and tangential direction $F_{\mathrm{t}}$ as shown in Fig.3 could thus be obtained

$$
\left\{\begin{array}{l}
F_{\mathrm{r}}=\iint P \cos \theta \cdot r d \theta d \xi \\
F_{\mathrm{t}}=\iint P \sin \theta \cdot r d \theta d \xi
\end{array}\right.
$$

Then the non-dimensional load-carrying capacity $\zeta$ and the attitude angle $\phi$ can be expressed as

$$
\zeta=\sqrt{F_{\mathrm{r}}^{2}+F_{\mathrm{t}}^{2}}, \phi=\arctan \frac{F_{\mathrm{t}}}{F_{\mathrm{r}}}
$$

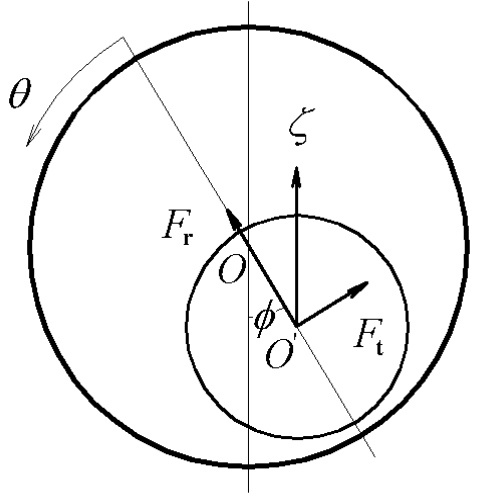

Figure 3. The force component of the micro gas bearing.

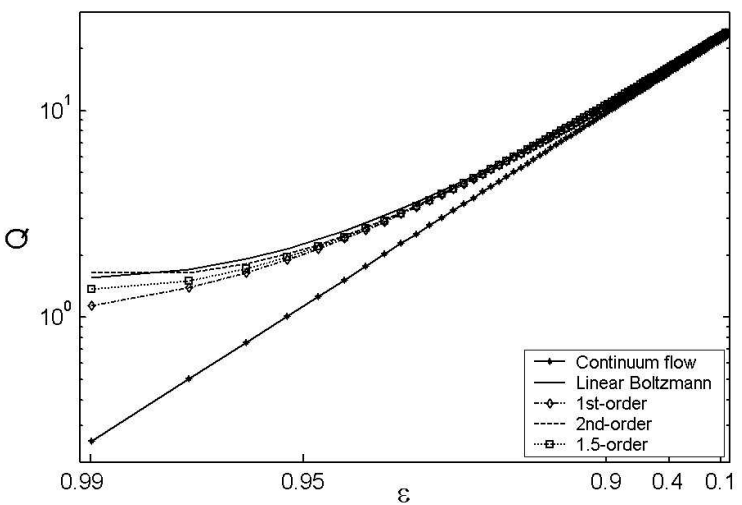

Figure 4. Comparison of non-dimensional flow rate

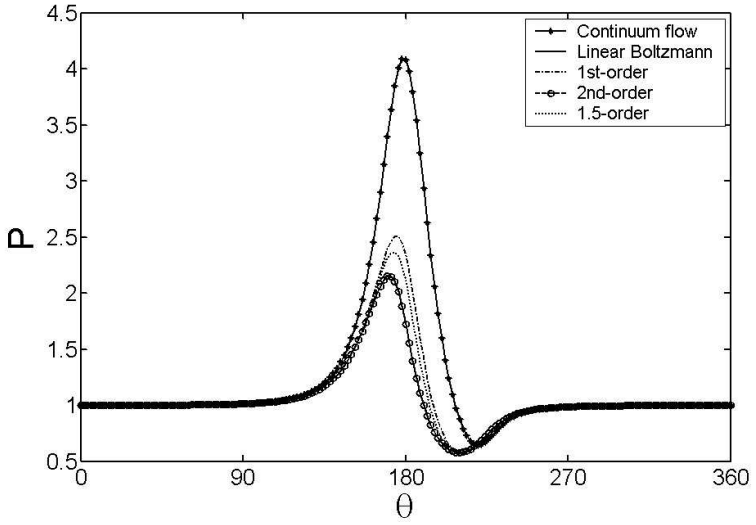

Figure 5. Comparison of the gas pressure.

For the micro gas bearing presented in this discussion, the non-dimensional flow rate is compared in Fig. 4 where the eccentricity ratio ranges from 0.1 to 0.99 . It can be seen that the second order slip model excels others in the sense of approximation to the linearized Boltzmann equation in the overall operational range. When the eccentricity ratio is less than 0.9 , the result of the first order slip model is also acceptable. Figure 5 depicts the gas pressure distributions of the gas film when the eccentricity 
ratio is 0.95 and the bearing number is 5 . It is noted that results of the second order slip model are almost the same as that of the linearized Boltzmann equation, while the first order and the 1.5 order slip models overestimate the gas pressure. Consequently, the load capacities are overestimated and the attitude angles are underestimated, as shown in Fig.6 and Fig.7 respectively.

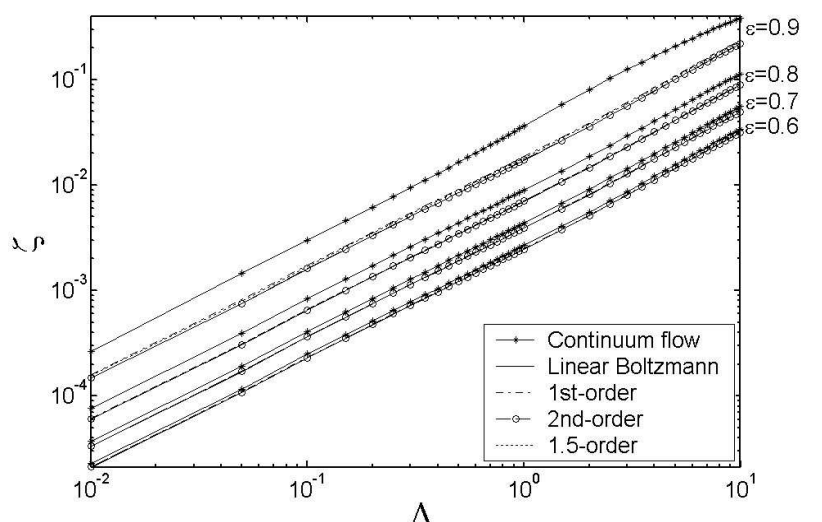

Figure 6. Comparison of the load carrying capacity

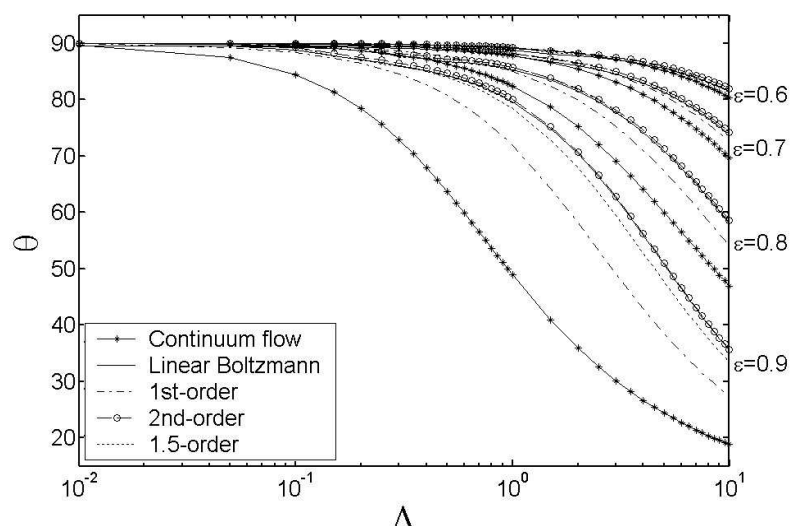

Figure 7. Comparison of the attitude angle.

\section{Motion equations and the linear threshold speed}

In this discussion, it is assumed that the micro rotor-bearing system is comprised of a rigid rotor and rigid mounted bushing. Also, the rotor is perfectly co-axial with the bushing. Thus, the motion equations of the rotor in the Cartesian coordinates can be written as follows:

$$
\left.\begin{array}{l}
m \ddot{x}=-f_{x}+f_{L}+m e_{m} \omega^{2} \cos (\theta+\phi) \\
m \ddot{y}=-f_{y}+m e_{m} \omega^{2} \sin (\theta+\phi)
\end{array}\right\}
$$

where $x$ and $y$ are the projection of the rotor displacement onto the $\mathrm{X}$-axis and Y-axis. Likewise, $f_{x}$ and $f_{y}$ are components of gas-film force in these two directions.

Eq. (20) can be rewritten in state space form as follows

$$
\left.\begin{array}{l}
Z_{1}^{\prime}=Z_{3} \\
Z_{2}^{\prime}=Z_{4} \\
Z_{3}^{\prime}=\frac{-F_{x}+F_{L}}{M \Lambda^{2}}+\beta \sin (\omega t+\phi) \\
Z_{4}^{\prime}=\frac{-F_{y}}{M \Lambda^{2}}+\beta \cos (\omega t+\phi)
\end{array}\right\}
$$

where $Z_{1}=X, Z_{2}=Y, Z_{3}=X^{\prime}, Z_{4}=Y^{\prime}$ are the state variables of the system. And some dimensionless variables are as follows

$$
X=\frac{x}{C}, Y=\frac{y}{C}, \beta=\frac{e_{m}}{C}, \tau=\omega t, M=\frac{m \cdot P_{a}}{72 \mu^{2} L} \psi^{5}, F=\frac{f}{2 R L P_{a}}
$$


The gas-film forces are nonlinear function and can be secured by solving the Reynolds equation with FDM. However, in most practical calculations, it is assumed that perturbation of the rotor from its initial position is small enough that the gas-film forces could be considered as linear. Thus the gas-film force increments are modeled by a first-order Taylor expansion ${ }^{[15,16]}$ as follows

$$
\left.\begin{array}{l}
\Delta F_{x}=K_{x x} \Delta Z_{1}+K_{x y} \Delta Z_{2}+D_{x x} \Delta Z_{3}+D_{x y} \Delta Z_{4} \\
\Delta F_{y}=K_{y x} \Delta Z_{1}+K_{y y} \Delta Z_{2}+D_{y x} \Delta Z_{3}+D_{y y} \Delta Z_{4}
\end{array}\right\}
$$

where $\Delta F_{x}$ and $\Delta F_{y}$ are the bearing force increments, $K_{i j}$ and $D_{i j}$ the dimensionless stiffness and damping coefficient respectively. Substituting Eqs. (23) into Eqs. (21), and assume that the unbalance ratio is zero $(\beta=0)$, the system's free vibration equations can be derived in matrix form:

$$
\left(\begin{array}{l}
\Delta Z_{1}^{\prime} \\
\Delta Z_{2}^{\prime} \\
\Delta Z_{3}^{\prime} \\
\Delta Z_{4}^{\prime}
\end{array}\right)=\frac{1}{M \Lambda^{2}}\left[\begin{array}{cccc}
0 & 0 & M \Lambda^{2} & 0 \\
0 & 0 & 0 & M \Lambda^{2} \\
-K_{x x} & -K_{x y} & -D_{x x} & -D_{x y} \\
-K_{y x} & -K_{y y} & -D_{y x} & -D_{y y}
\end{array}\right] \cdot\left(\begin{array}{l}
\Delta Z_{1} \\
\Delta Z_{2} \\
\Delta Z_{3} \\
\Delta Z_{4}
\end{array}\right)=\mathbf{A} \cdot\left(\begin{array}{l}
\Delta Z_{1} \\
\Delta Z_{2} \\
\Delta Z_{3} \\
\Delta Z_{4}
\end{array}\right)
$$

Subject matrix A to eigenvalue evaluation so as to investigate the linear dynamic stability of the system. The real part of each eigenvalue, $\delta$, is the damping rate and corresponds to how quickly a perturbation of the rotor decays. $\delta<0$ implies a decay motion (stable), while $\delta>0$ implies a diverging motion (unstable). There are four eigenvalues of matrix A . We only consider the one with the biggest positive real part, because it represents the most dangerous mode and can be observed in an experimental measurement. At the threshold of instability, the matrix $\mathbf{A}$ has an eigenvalue with real part equal to zero, and the following equations can be derived

$$
\left.\begin{array}{c}
K_{e q}=M \Lambda^{2} \cdot \gamma^{2} \\
K_{e q}=\frac{K_{x x} D_{y y}+K_{y y} D_{x x}-K_{x y} D_{y x}-K_{y x} D_{x y}}{D_{x x}+D_{y y}} \\
\gamma^{2}=\frac{\left(K_{e q}-K_{x x}\right)\left(K_{e q}-K_{y y}\right)-K_{x y} K_{y x}}{D_{x x} D_{y y}-D_{x y} D_{y x}}
\end{array}\right\}
$$

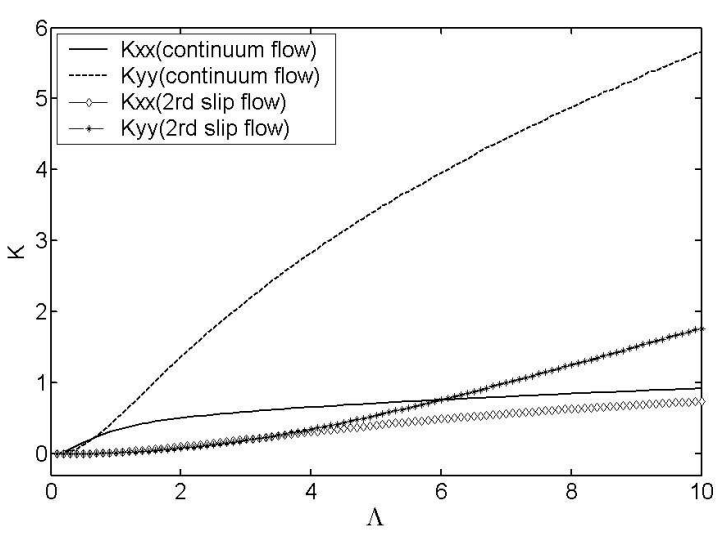

(a) Stiffness coefficient

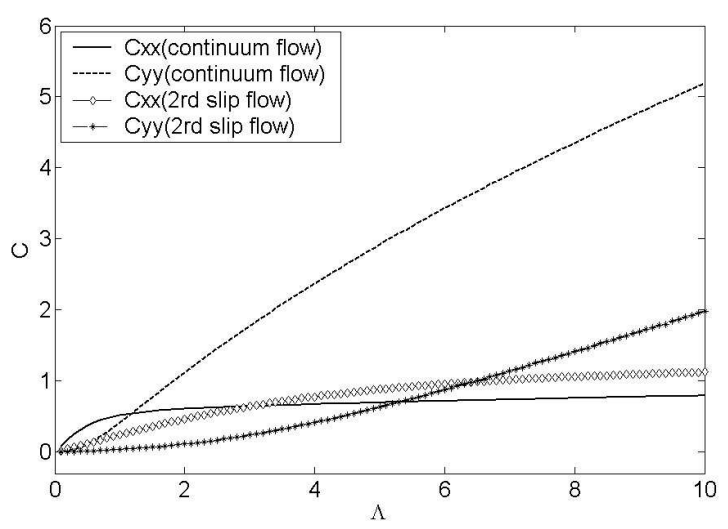

(b) Damping coefficient

Figure 8. The comparison of the dynamic coefficients at $\varepsilon=0.9$. 
Figure 8 presents the comparison of the stiffness and damping coefficients between the continuum model and second order slip model when the eccentricity ratio is 0.9. It can be seen that the dynamic coefficients increase with the increase of the bearing number, indicating the enhancement of the stability of the micro rotor system.

Referring to Fig. 8, the dynamic coefficients of the second order slip flow are much lower than those of the continuum flow. From the discussion in section 3, it is known that the second order slip flow predicts more accurate solutions for the micro gas bearing, and the continuum flow assumption lead to mistaken results. Furthermore, it is implied that the continuum flow assumption will underestimate the threshold speed for stable operation. Combining Eq.(25) and Eq.(26) with results shown in Fig.8, the threshold speed can be obtained. For the continuum flow, the stable threshold speed of the bearing system is close to the point $\Lambda=0.3$ when the eccentricity is 0.9 . Considering the slip effect, the threshold speed raise to $\Lambda=6.3$.

\section{Dynamic responses of the rotor-bearing system}
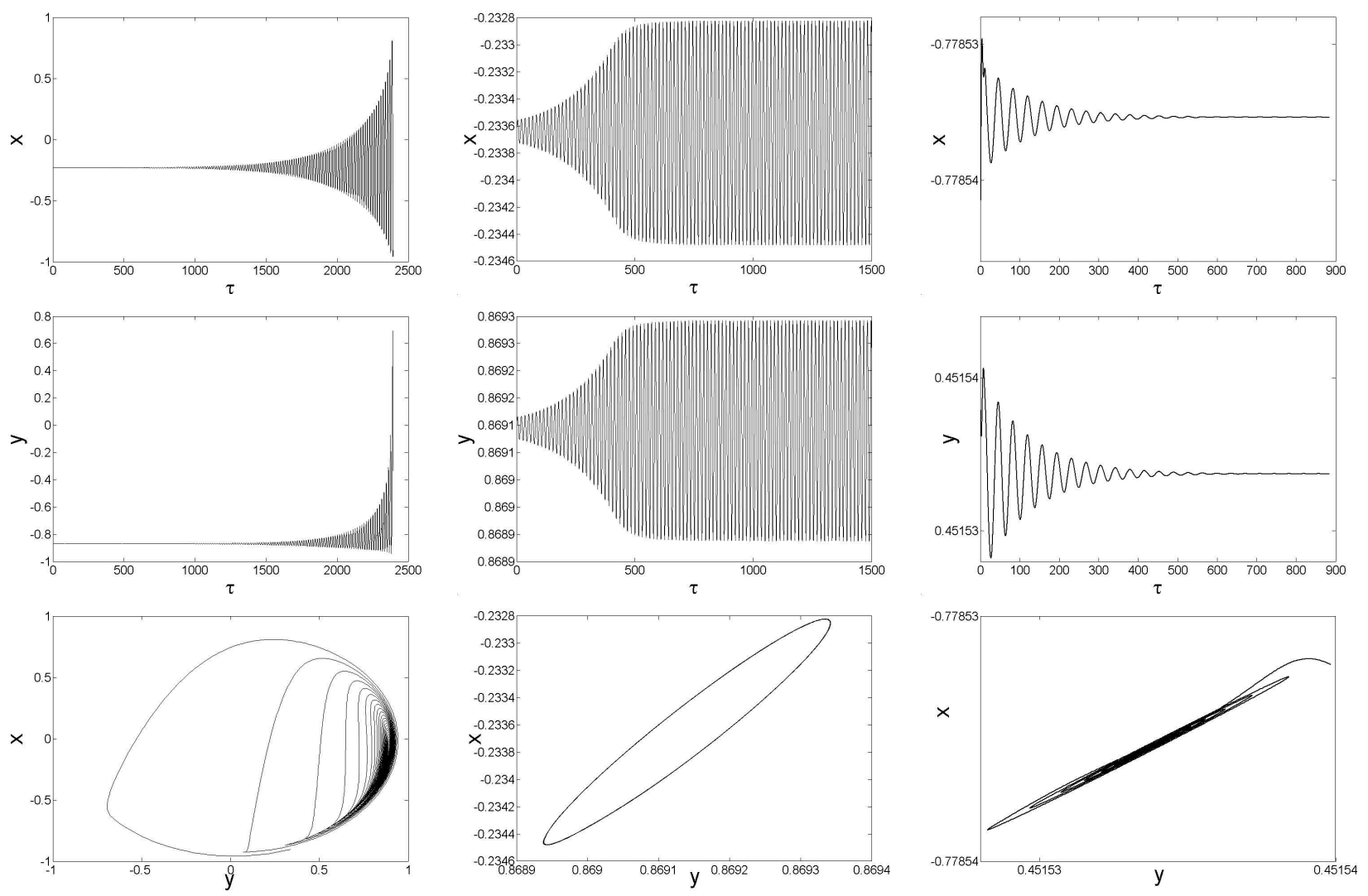

(a) $\Lambda=0.28$

(b) $\Lambda=0.3$

(c) $\Lambda=1.5$

Figure 9. The response of the rotor at different bearing number (continuum flow).

The numerical simulation of the rotor dynamical response can be achieved by solving the Reynolds equation and the motion equation subsequently. Eq. (21) for dynamical motion can be solved by fourth-order Rouge-Kutta method, and the displacements and velocities time series for rotor can thus be obtained. 
Given a slight disturbance, a balanced rotor will converge to its static equilibrium position or whirl around the static equilibrium if the rotational speeds is above the threshold speed. And it will crash into the bushing when the rotational speed is less than the threshold speed. With the continuum model assumption, the dynamic responses of the micro gas bearing at different rotational speed are shown in Fig.9 respectively. When the second slip model is adopted, the responses are different from the continuum flow case, as shown in Fig. 10.

By comparison between Fig.9 and Fig.10, it can be seen that not only the threshold speed of the stable operation is raised, but also the dynamical responses are different when employing the secondorder slip model. It is found that due to the slip effect, the micro rotor needs much more time to reach a stable operation or to crash into the bushing. Furthermore, the oscillation period of the whirl motion is prolonged when the second slip flow model is adopted. As a result, the whirl frequency is reduced due to the slip effect, as shown in Fig.11.
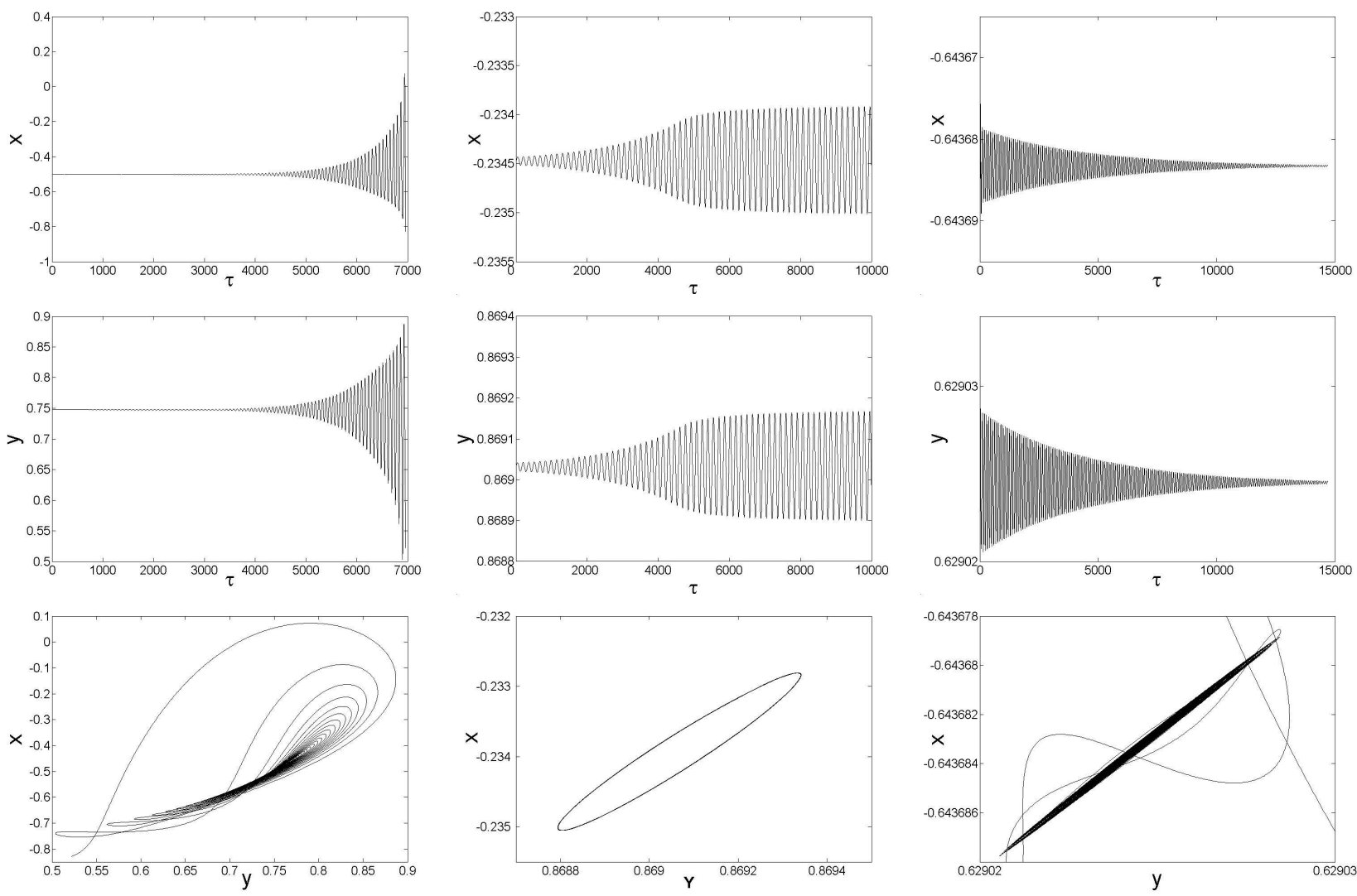

(a) $\Lambda=5$

(b) $\Lambda=6.5$

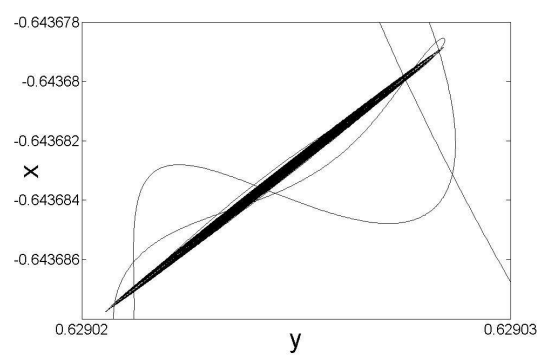

(c) $\Lambda=8$

Figure 10. The response of the rotor at different bearing number ( $2^{\text {nd }}$ slip flow $)$. 


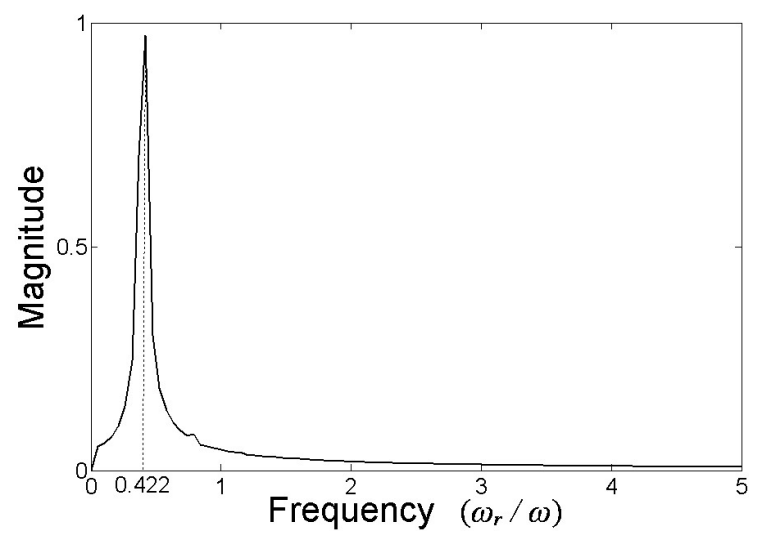

(a) continuum flow

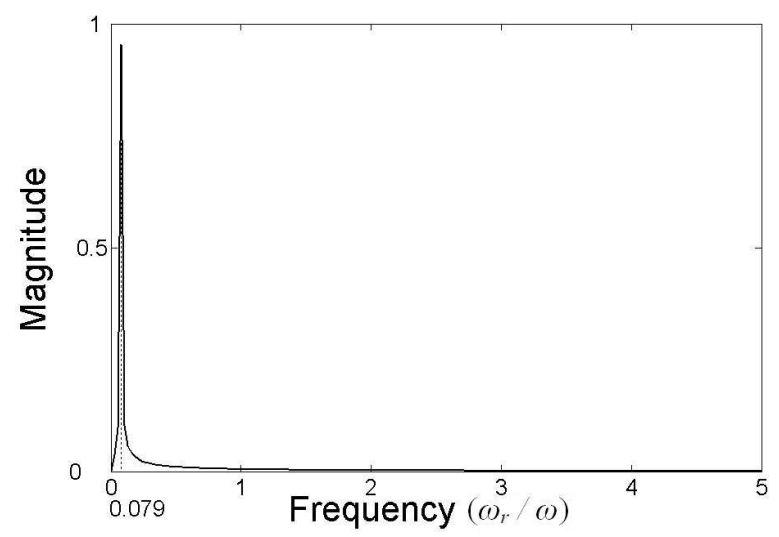

(b) second-order slip flow

Figure 11. The whirl frequency spectrum of the micro rotor.

In this discussion, the whirl is caused by the gas film force. It can be seen from Fig.11 that the whirl frequency is decreased due to the slip effect. When the continuum flow assumption is taken, the whirl frequency ratio is 0.422 . While, the ratio decreases to 0.079 for second order slip flow model. According to the analysis in the Section 3, the gas velocity at the rotor surface is subdued for the slip effect, and it equivalent to the case that a much slow rotating rotor supported by a gas bearing in which the continuum model govern the gas flow. Thus, the whirl frequency of the former is reduced. Consequently, the whirl frequency ratio is decreased.

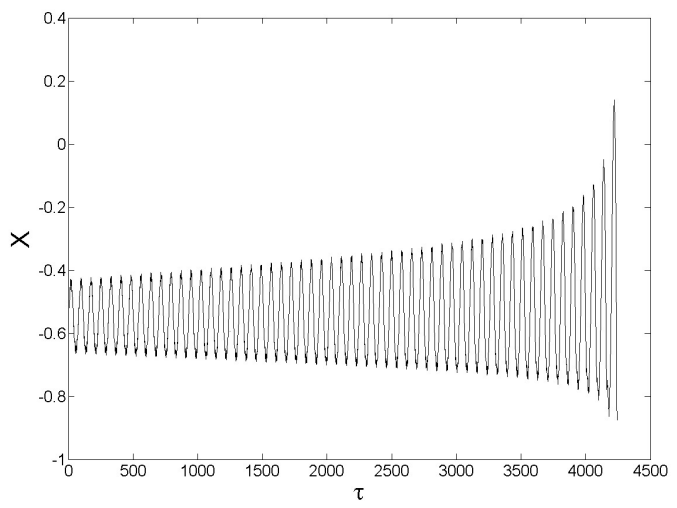

(a) The rotor center's displacement in X direction

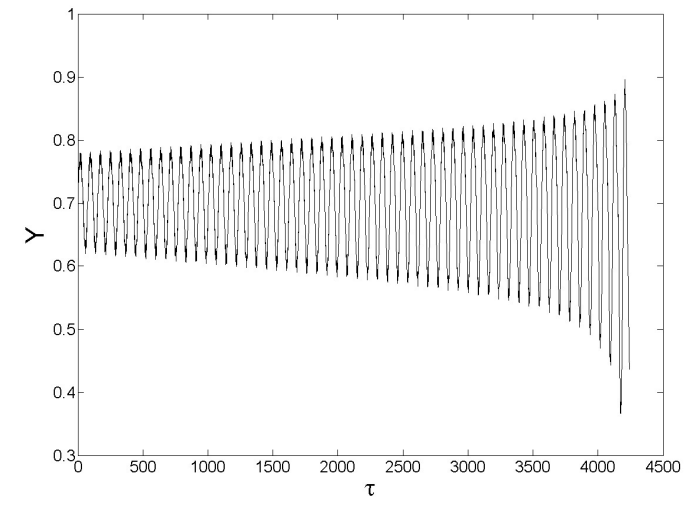

(b) The rotor center's displacement in Y direction

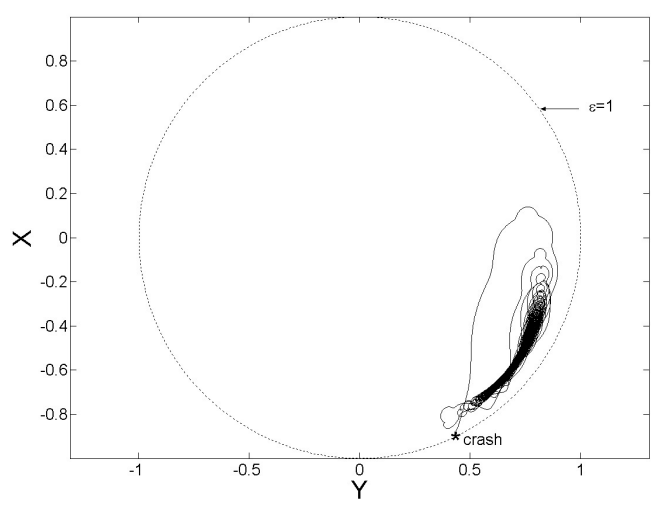

(c) The rotor center's orbit

Figure 12. The dynamical response of an unbalanced rotor $\left(\Lambda=6.0, \beta=1 \%, 2^{\text {nd }}\right.$ slip flow). 


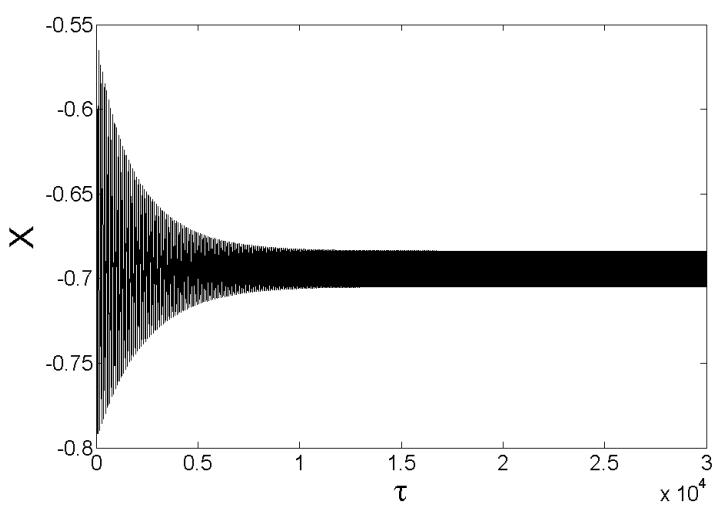

(a) The rotor center's displacement in $\mathrm{X}$ direction

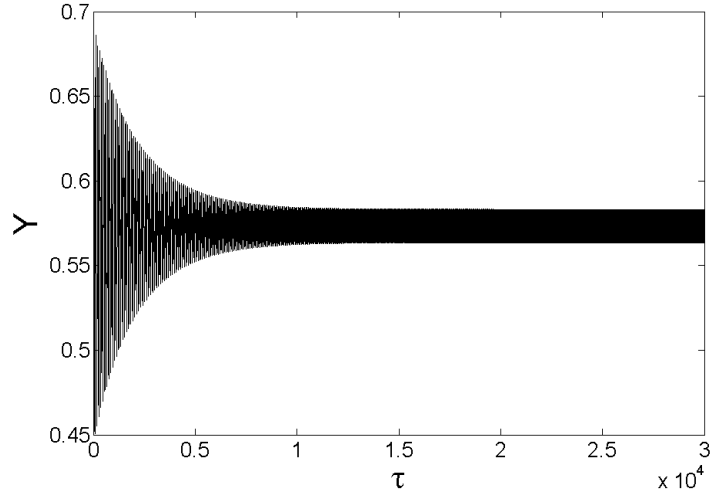

(b) The rotor center's displacement in Y direction

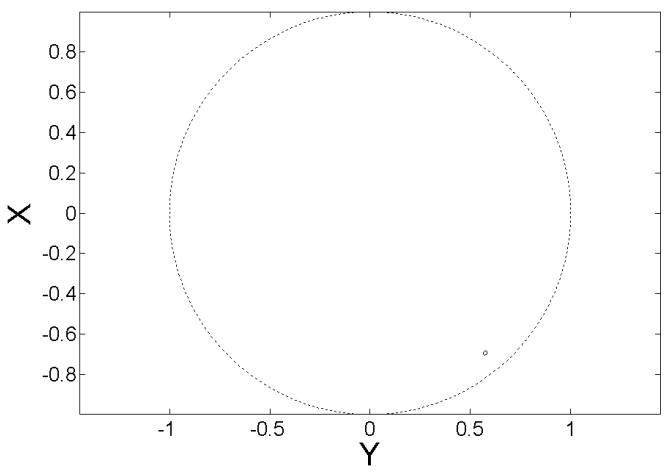

(c) The rotor center's orbit

Figure13. The dynamical response of an unbalanced rotor $\left(\Lambda=10, \beta=1 \%, 2^{\text {nd }}\right.$ slip flow $)$.

For a practical rotor, due to the fabrication defect, the mass center would inevitably depart from the geometry center, and the rotor is unbalanced. From the above discussion, it is known that the continuum flow assumption will lead to a mistaken result of the dynamic characteristics of the system. Thus, the second order slip flow is adopted for the dynamic analysis of the unbalanced micro rotor. For difference rotational speed, the dynamical responses of an unbalance motor are acquired, which are shown in Fig.12 and Fig.13.

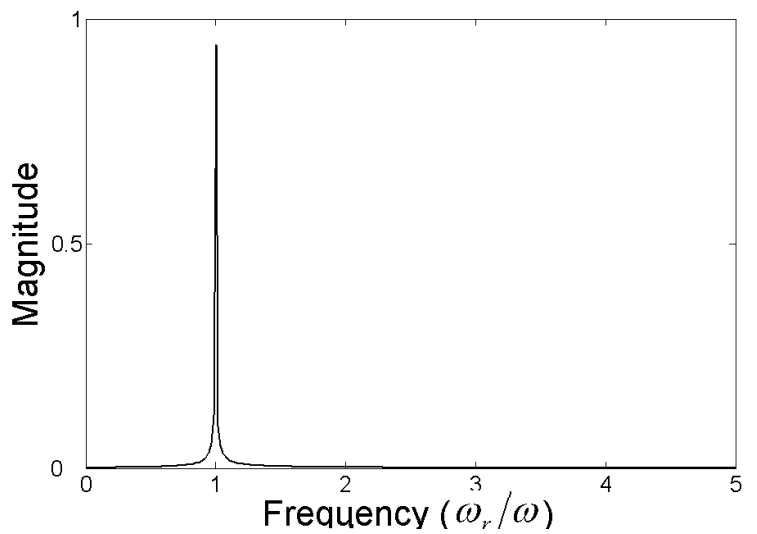

Figure 14. The frequency spectrum of an unbalanced rotor whirling motion $(\Lambda=10, \beta=1 \%$,

$$
2^{\text {nd }} \text { slip .flow) }
$$


For some unbalanced rotor, the oscillation amplitude will keep growing and the rotor will crash into the bushing $(\Lambda=6.0, \beta=1 \%)$, as shown in Fig.12. In other condition, the oscillation amplitude will decay to a certain value and the rotor motion become a whirl of small amplitude $(\Lambda=10, \beta=1 \%)$, as shown in Fig.13. Unlike balanced rotor, the unbalanced rotor will not converge to the static equilibrium position, for the unbalanced mass will be an excitation force due to the rotation of the rotor. For this reason, the whirl frequency is equal to the rotational rate as shown in Fig.14.

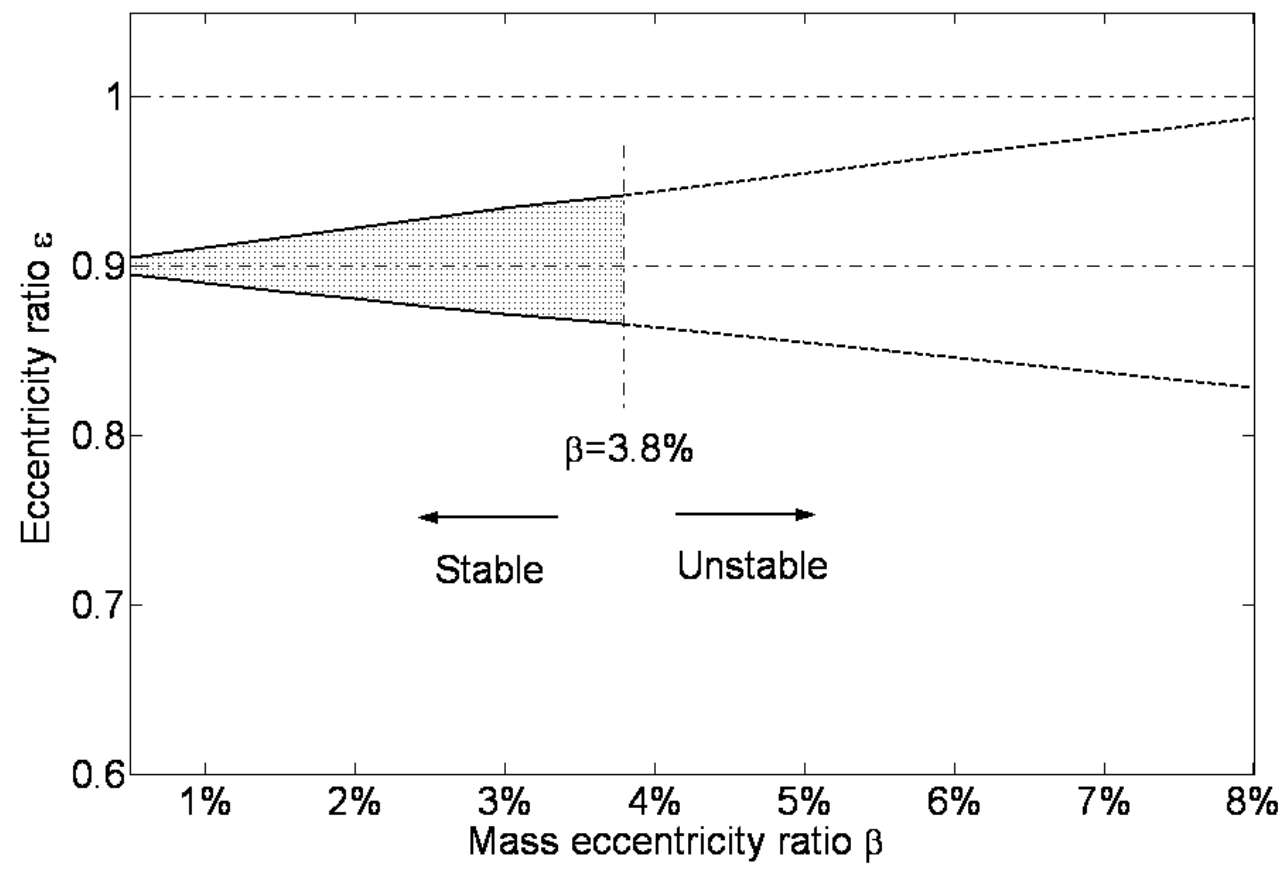

Figure 15. The maximum and the minimum eccentricity ratio of the rotor during the whirling motion versus the unbalance ratio ( $\Lambda=10$, second slip flow model).

With the increase of the unbalance ratio, the whirl amplitude increases, as shown in Fig. 15. The two lines in the figure represent the maximum and minimum eccentricity ratio of the rotor in the whirling motion. It shows that, when the unbalance ratio increases, the whirl amplitude is enlarged. And it is almost a linear relation. Deduced from the increase trend, it is supposed that the maximum eccentricity ratio of the rotor will approach 1 when the unbalance ratio is about $10 \%$, at which the rotor will crash into the bushing. But during the calculation, it is found that the rotor will crash into the bushing when the unbalance ratio is $3.8 \%$, which is much less than $10 \%$. Examining the dynamical response shown in Fig.13, it is found that the whirl amplitude of the unsteady state oscillation is much lager than that of the steady state motion. Consequently, the maximum eccentricity ratio of the rotor at the unsteady state is much higher. As a result, the rotor will crash into the bushing before steady whirl state when the unbalance ratio is $3.8 \%$.

\section{Conclusions}

In this study, the performances of the micro gas bearing are presented. Besides, the stability and the dynamical response of the micro rotor supported by the micro gas bearing are discussed. Considering the micro length scale and the micro fabrication constraints, the driving flow mechanisms in the micro 
gas bearing and the rotor dynamic characteristics of the bearing system are different from those of conventional gas bearings. From the analysis and the discussion, some conclusions could be drawn:

(1) In most operation conditions of the micro gas bearing presented in the paper, the second order slip model predicts a good approximation to the linearized Boltzmann equation;

(2) The slip effect at the solid boundary increases the flow rate of the gas flow. Hence, the gas pressure of the lubricated gas film is decreased, and the load carrying capacity of the micro gas bearing is reduced.

(3) The dynamic coefficients of a micro rotor-bearing system are decreased due to the slip effect, which means that the system stability is compromised. Therefore, the micro rotor must operate at much higher speed to keep stable.

(4) When subject to a slight disturbance, a balance micro rotor will converge to or whirl around the static equilibrium position when the rotational speed is not less than the threshold speed. Otherwise the disturbance will be enhanced and the rotor will crash into the bushing.

(5) Considering the slip effect, the rotor's whirl frequency is decreased, and the micro rotor needs more time to reach a stable operation or crash into the bushing.

(6) For an unbalanced micro rotor, the rotor would not converge to the static equilibrium position. The stable operation is a slight whirl which is excited by the unbalance force. The whirl frequency is equal to the rotational frequency.

(7) The whirl amplitude increases with the increase of the unbalance ratio. And the rotor would not stay stable when the unbalance ratio is too big.

\section{Acknowledgements}

This work was supported by the the National Outstanding Youth Foundation of China under Grant No.10325209.

\section{References and Notes}

1. Fréchette, L. G.; Jacobson, S. A.; Breuer, K. S.; Ehrich, F. F.; Ghodssi, R.; Khanna, R.; Wong, C. W.; Zhang, X.; Schmidt, M. A.; Epstein, A. H. High-speed microfabricated silicon turbomachinery and fluid film bearings. Journal of Microelectromechanical Systems, 2005, 14 (1), 141-152.

2. Ehrich, F. F.; Jacobson, S. A. Development of high-speed gas bearings for high-power density microdevices. Journal of Engineering for Gas Turbines and Power, 2003, 125 (1), 141.

3. Zhang, W. M.; Meng, G. Contact dynamics between the rotor and bearing hub in an electrostatic micromotor. Microsystem Technologies-Micro-and Nanosystems-Information Storage and Processing Systems, 2005, 11 (6), 438-443.

4. G. Meng; W. M. Zhang; H. Huang; H. G. Li; Chen, D. Micro-rotor dynamics for micro-electromechanical systems. Chaos, Solitons \& Fractals, 2006,

5. Kenneth Breuer, F. E., Luc Fréchette, Stuart Jacobson, C-C Lin, D J Orr, Edward Piekos, Nicholas Savoulides, Chee-Wei Wong. Challenges for lubrication in high speed MEMS. NanoTribology, 2001, 
6. Savoulides, N.; Breuer, N. S.; Jacobson, S. Low-Order Models for Very Short Hybrid Gas Bearings. Journal of Tribology, 2001, 123 (2), 368-375.

7. Wang, C. C.; Chen, C. K. Bifurcation analysis of self-acting gas journal bearings. Journal of Tribology, 2001, 123 (4), 755-767.

8. Burgdorfer, A. The influence of the molecular mean free path on the performance of hydrodynamic gas lubricated bearings. ASME Journal of Basic Engineering, 1959, 81 (1), 94100 .

9. Hsia, Y. T.; Domoto, G. A. An Experimental Investigation Of Molecular Rarefaction Effects In Gas Lubricated Bearings At Ultralow Clearances. Journal Of Lubrication TechnologyTransactions Of The Asme, 1983, 105 (1), 120-130.

10. Mitsuya, Y. Modified Reynolds-Equation For Ultra-Thin Film Gas Lubrication Using 1.5-Order Slip-Flow Model And Considering Surface Accommodation Coefficient. Journal of TribologyTransactions of the Asme, 1993, 115 (2), 289-294.

11. Huang, H.; Meng, G.; Zhao, S. Steady performance of a micro gas-lubricated bearing. Proceedings of STLE/ASME International Joint Tribology Conference, San Antonio, TX, United States, 2006, 7-14.

12. Sun, Y.; Chan, W. K.; Liu, N. A slip model with molecular dynamics. Journal of Micromechanics and Microengineering, 2002, 12 (3), 316-322.

13. Bird, G. A. Molecular Gas Dynamics and the direct simulation of gas flows. New York, NY: Oxford Science Publications, 1994.

14. Cercignani, C.; Daneri, A. Variational approach to boundary-value problems in kinetic theory. Journal of Applied Physics, 1966, 6, 1167-1173.

15. Lund, J. Review of the concept for dynamic coefficients for fluid film journal bearings. journal of Tribology, 1987, 109, 37-41.

16. Zhao, S. X.; Xu, H.; Meng, G.; Zhu, J. Stability and response analysis of symmetrical single-disk flexible rotor-bearing system. Tribology International, 2005, 38 (8), 749-756.

(C) 2007 by MDPI (http://www.mdpi.org). Reproduction is permitted for noncommercial purposes. 